\title{
Near-Optimal, Dynamic Module Reconfiguration in a Photovoltaic System to Combat Partial Shading Effects
}

\author{
Xue Lin ${ }^{1}$, Yanzhi Wang ${ }^{1}$, Siyu Yue ${ }^{1}$, Donghwa Shin ${ }^{2}$, Naehyuck Chang ${ }^{2}$, and Massoud Pedram ${ }^{1}$ \\ ${ }^{1}$ Department of Electrical Engineering, University of Southern California, Los Angeles, CA, USA
}

${ }^{2}$ Seoul National University, Seoul, Korea

\{xuelin, yanzhiwa, siyuyue\}@usc.edu, \{dhshin, naehyuck\}@elpl.snu.ac.kr, pedram@usc.edu

\begin{abstract}
Partial shading is a serious obstacle to effective utilization of photovoltaic (PV) systems since it can result in a significant output power degradation for the system. A PV system is organized as a series connection of PV modules, each module comprising of a number of series-parallel connected cells. This paper presents modified PV cell structures with integrated switches, imbalanced cell connection topologies for PV modules, and a dynamic programming algorithm to produce near-optimal reconfigurations of each PV module with the goal of maximizing the system output power level under any partial shading patterns. Through simulations, we have demonstrated up to a factor of $2.3 \mathrm{X}$ improvement in the output power level of a PV system comprised of $3 \mathrm{PV}$ modules with $60 \mathrm{PV}$ cells per module.
\end{abstract}

\section{Categories and Subject Descriptors}

B.8.2 [Performance and Reliability]: Performance Analysis and Design Aids.

\section{General Terms}

Algorithms, Management, Performance, Design.

\section{Keywords}

Photovoltaic System, Partial Shading, Photovoltaic Module Reconfiguration, Dynamic Programming.

\section{INTRODUCTION}

Due to increasing appetite for energy sources and environmental concerns about fossil fuels, there has been a growing demand for renewable energy resources, which are clean and eco-friendly (pollution-free.) Among renewable resources, photovoltaic (PV) energy generation techniques have received significant attention, since solar energy is abundant, and can be easily scaled up. Thanks to extensive research efforts on PV energy generation technologies, various scales of PV-powered energy generation systems (PV systems) have been deployed for practical applications, such as PV power stations, solar-powered vehicles, and solar power heating and lighting appliances.

Like most other renewable energy systems, PV systems have a major weakness in that their power output level is greatly affected by environmental conditions. More precisely, solar irradiation received by a PV module is changing frequently, according to the time of day and weather conditions (e.g., a passing cloud.) To

Permission to make digital or hard copies of all or part of this work for personal or classroom use is granted without fee provided that copies are not made or distributed for profit or commercial advantage and that copies bear this notice and the full citation on the first page. To copy otherwise, to republish, to post on servers or to redistribute to lists, requires prior specific permission and/or a fee.

DAC 2012, June 3-7, 2012, San Francisco, California, USA

Copyright 2012 ACM 978-1-4503-1199-1/12/06 ..\$10.00 provide a rather steady power output level, standalone PV systems are equipped with electrical energy storage (EES) elements. Furthermore, PV modules exhibit highly non-linear currentvoltage (I-V) characteristics that change with solar irradiance. Therefore, a maximum power point tracking (MPPT) technique is mandated to extract maximum power from PV modules [1][2]. Recently, the maximum power transfer tracking (MPTT) method, which accounts for changes in the efficiency of the charger, can be more effective than the MPPT methods [3][4].

In a PV system, a string of PV modules (i.e., a PV string) are connected in series to produce a desired voltage level, which is then fed to a charger. This architecture reduces the cost of the PV system due to sharing of the charger among PV modules. We call such a structure the string charger interface. Solar irradiations received by PV cells in the PV system, may be different, and such a phenomenon is known as partial shading. Unfortunately, the string charger interface is vulnerable to partial shading, which not only reduces the maximum output power of the shaded PV cell itself, but also makes non-shaded PV cells that are in series with the shaded one deviate from their MPPs ${ }^{1}$. This makes the maximum output power of a PV module string much lower than the sum of the MPP power values of all PV cells in the string. Partial shading may also result in multiple power peaks in the power-voltage $(\mathrm{P}-\mathrm{V})$ characteristics of a PV string. Therefore the MPPT techniques must be modified to track a global optimum operating point instead of a local optimum [5][6] because the unimodality assumption of the PV string P-V characteristics is the basis for conventional MPPT techniques. But modified MPPT techniques increase the complexity of the PV system controller, while non-shaded PV cells still suffer from power loss due to the deviation from their MPPs caused by shaded cells.

PV module reconfiguration techniques, which have the potential of exploiting MPPs of both non-shaded and shaded PV cells in a partially shaded PV string, may maintain the power output level of PV system under partial shading. Various PV reconfiguration techniques have been proposed [7] [9]. However, they suffer from one or more of the following limitations.

(1) To compensate the power loss from shaded PV cells, (many) extra PV cells are needed for performing reconfiguration.

(2) There is a lack of systematic and scalable structural support or effective control mechanism.

(3) Variations in the efficiency of the charger or inverter are overlooked, which may result in a sizeable degradation in the system energy conversion efficiency.

(4) The PV system employs an individual charger interface, in which each PV module has an individual charger for

\footnotetext{
${ }^{1}$ MPP stands for maximum power point. On I-V characteristics of a PV cell/module/string, there is a point $(V, I)$ where the power is maximized. This point is the MPP of a PV cell/ module/string.
} 
operating point setting, thereby, increasing the hardware cost of a PV system.

In this paper, we present a dynamic imbalanced PV module reconfiguration method with both a scalable structural support, as well as a systematic and near-optimal control algorithm, to overcome the power output degradation in a PV system under partial shading. We realize imbalanced reconfiguration for PV modules. Different from [10], which always maintains an $n \times m$ configuration for the PV module (where $m$ is the number of parallel-connected elements in each $P V$ group ${ }^{2}$ and $n$ is the number of groups that are connected in series), our imbalanced reconfiguration method allows more flexible PV module configurations in which there can be an arbitrary number of PV groups connected in series in the PV module and PV groups may have different numbers of parallel-connected PV cells. Our reconfiguration method can utilize both non-shaded and shaded PV cells in a PV module to the largest extent under partial shading.

We present the imbalanced reconfiguration method on the PV system with the more widely-used and cost-effective string charger interface. We introduce an effective reconfiguration control algorithm to realize adaptive and near-optimal PV module reconfiguration for each PV module according to partial shading pattern and charger efficiency variation. The proposed control mechanism is based on dynamic programming with polynomial time complexity. Therefore, it can be incorporated into PV systems with negligible extra computational overhead. Experimental results demonstrate that our proposed reconfiguration method can result in up to a $2.3 \mathrm{X}$ output power level improvement, compared with the baseline PV system without PV module reconfiguration method.

\section{COMPONENT MODELS}

\subsection{PV Cell Model and Characterization}

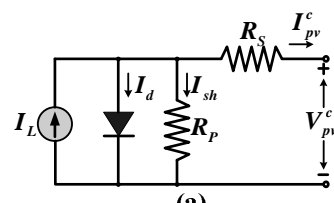

(a)

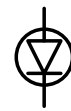

(b)
Figure 1. (a) Equivalent circuit and (b) symbol of a PV cell.

We use $V_{p v}^{c}$ and $I_{p v}^{c}$ to denote the output voltage and current of a PV cell, respectively. Figure 1(a) shows PV cell equivalent circuit model, with I-V characteristics given by

$I_{p v}^{c}=I_{L}-I_{d}-I_{s h}$

$$
=I_{L}(G)-I_{0}(T)\left(e^{\left(V_{p v}^{c}+I_{p v}^{c} \cdot R_{S}\right) \cdot \frac{q}{A k T}}-1\right)-\frac{V_{p v}^{c}+I_{p v}^{c} \cdot R_{S}}{R_{p}},
$$

where

$$
I_{L}(G)=\frac{G}{G_{S T C}} \cdot I_{L}\left(G_{S T C}\right)
$$

and

$$
I_{0}(T)=I_{0}\left(T_{S T C}\right) \cdot\left(\frac{T}{T_{S T C}}\right)^{3} \cdot e^{\frac{q E g}{A k} \cdot\left(\frac{1}{T_{S T C}}-\frac{1}{T}\right)} .
$$

For parameters in (1) (3), $G$ is irradiance level; $T$ is cell temperature; $q$ is charge of the electron; $E_{g}$ is bandgap and $k$ is Boltzmann's constant. STC stands for standard test condition where $G_{S T C}=1000 \mathrm{~W} / \mathrm{m}^{2}$ and $T_{S T C}=25^{\circ} \mathrm{C}$. For rest parameters, i.e., photo-generated current at STC $I_{L}\left(G_{S T C}\right)$, dark saturation current at STC $I_{0}\left(T_{S T C}\right), \mathrm{PV}$ cell series resistance $R_{S}, \mathrm{PV}$ cell

\footnotetext{
${ }^{2}$ A PV module consists of series-connected PV groups. A PV group composes of a number of parallel-connected PV cells.
}

parallel (shunt) resistance $R_{p}$, and diode ideality factor $A$, we adopt the method in [11] to extract their values. Therefore, based on this PV cell model, we can obtain I-V characteristics of a PV cell under any given environmental condition $(G, T)$.

\subsection{Charger Model}

Figure 2 shows the model of a pulse width modulation buck-boost switching converter, which is used as the charger in PV system [12]. The input ports of the charger are connected to the PV string. The output ports are connected to the load. Then the operating point of the PV module string can be regulated by the charger through controlling its output current. The input voltage, input current, output voltage and output current of the charger are denoted by $V_{\text {in }}, I_{\text {in }}, V_{\text {out }}$, and $I_{\text {out }}$, respectively. The power loss of the charger $P_{\text {conv }}$ satisfies

$$
V_{\text {in }} \cdot I_{\text {in }}=P_{\text {conv }}+V_{\text {out }} \cdot I_{\text {out }} \text {. }
$$

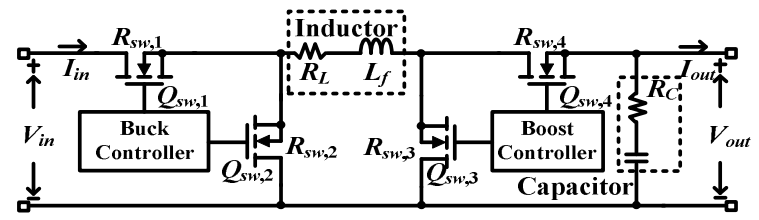

Figure 2. Buck-boost converter architecture.

When the charger is at the buck mode $\left(V_{\text {in }}>V_{\text {out }}\right)$, its power loss $P_{\text {conv }}$ is given by

$P_{\text {conv }}=I_{\text {out }}^{2} \cdot\left(R_{L}+D \cdot R_{s w, 1}+(1-D) \cdot R_{s w, 2}+R_{s w, 4}\right)$

$+\frac{(\Delta I)^{2}}{12} \cdot\left(R_{L}+D \cdot R_{s w, 1}+(1-D) \cdot R_{s w, 2}+R_{s w, 4}+R_{C}\right)$

$+V_{\text {in }} \cdot f_{s} \cdot\left(Q_{s w, 1}+Q_{s w, 2}\right)+V_{\text {in }} \cdot I_{\text {controller }}$,

where $D=V_{\text {out }} / V_{\text {in }}$ is the PWM duty ratio and $\Delta I=V_{\text {out }} \cdot(1-$ $D) /\left(L_{f} \cdot f_{s}\right)$ is the maximum current ripple; $f_{s}$ is the switching frequency; $I_{\text {controller }}$ is the current of the micro-controller of the charger; $R_{L}$ and $R_{C}$ are the internal series resistances of the inductor $L$ and the capacitor $C$, respectively; $R_{s w, i}$ and $Q_{s w, i}$ are the turn-on resistance and gate charge of the $i$-th MOSFET switch shown in Figure 2, respectively. The charger power loss $P_{\text {conv }}$ at the boost mode $\left(V_{\text {in }} \leq V_{\text {out }}\right)$ is given by

$$
\begin{aligned}
& P_{\text {conv }}=\left(\frac{I_{\text {out }}}{1-D}\right)^{2} \cdot\left(R_{L}+D \cdot R_{s w, 3}+(1-D) \cdot R_{s w, 4}+\right. \\
& \left.R_{s w, 1}+D \cdot(1-D) \cdot R_{C}\right)+\frac{(\Delta I)^{2}}{12}\left(R_{L}+D \cdot R_{s w, 3}+(1-\right. \\
& \left.D) R_{s w, 4}+R_{s w, 1}+(1-D) R_{C}\right)+V_{\text {out }} \cdot f_{s} \cdot\left(Q_{s w, 3}+\right. \\
& Q_{s w, 4}+V_{\text {in }} \cdot I_{\text {controller }},
\end{aligned}
$$

where $D=1-V_{\text {in }} / V_{\text {out }}$ and $\Delta I=V_{\text {in }} \cdot D /\left(L_{f} \cdot f_{s}\right)$. Moreover, we use $I_{\text {out }}=$ Chg_Out_I $\left(V_{\text {in }}, I_{\text {in }}, V_{\text {out }}\right)$ to denote the function which calculates the charger output current $I_{\text {out }}$, given its input voltage and current, as well as its output voltage.

\section{IMBALANCED PV CELL CONNECTION TOPOLOGY}

This paper proposes an imbalanced PV cell connection topology (imbalanced topology) for each PV module in the system. An $N$ cell PV module has $n$ series-connected PV groups. PV cells in a PV group are parallel-connected. The imbalanced topology is very flexible because $n$ could be any nonzero integer less than or equal to $N$. And the number of parallel-connected PV cells $m_{j}(>0)$ in the $j$-th $(1 \leq j \leq n)$ PV group satisfies

$$
\sum_{j=1}^{n} m_{j}=N \text {. }
$$


We denote an imbalanced topology or configuration of a PV module by $\mathcal{C}\left(n ; m_{1}, m_{2}, \cdots, m_{n}\right)$. Figure 3 shows an example imbalanced topology of $\mathcal{C}(3 ; 4,3,5)$.

Figure 4 shows the reconfigurable PV module architecture for implementing imbalanced topologies of a PV module. The architecture is already provided in [10]. However we attempt the imbalanced reconfiguration for the first time. Each PV cell is integrated with three switches, i.e., a series switch (S-switch) and two parallel switches (P-switches), except for the last one. The Pswitches connect PV cells in parallel, and the S-switches connect the PV groups in series. We denote $\mathrm{S}$-switch of the $i$-th PV cell by $S_{S, i}$, and the top and bottom P-switches of the $i$-th PV cell by $S_{P T, i}$ and $S_{P B, i}$, respectively. Both $S_{P T, i}$ and $S_{P B, i}$ must be closed or open together. $S_{P T, i}$ and $S_{P B, i}$ are closed exactly if $S_{S, i}$ is open, and vice versa. Refer to Figure 3 on how an imbalanced topology is implemented, in which the first PV group contains PV cells 1 to 4, and these four PV cells are connected in parallel by the P-switches of PV cells 1 to 3 . The first PV group is connected in series with the second PV group by the S-switch of PV cell 4.

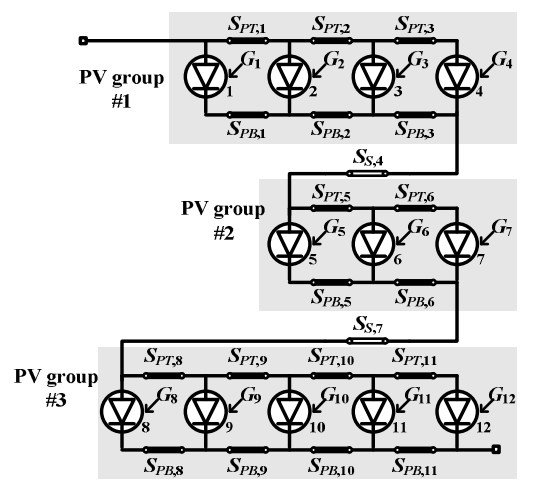

Figure 3. An imbalanced topology of a 12-cell PV module.

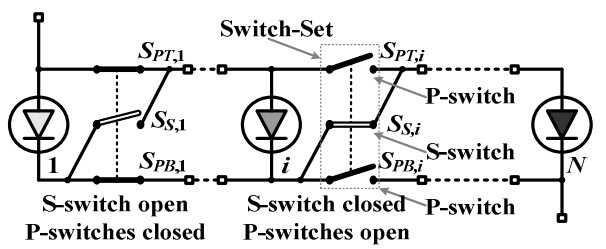

Figure 4. The $N$-cell reconfigurable PV module architecture.

An imbalanced configuration $\mathcal{C}\left(n ; m_{1}, m_{2}, \cdots, m_{n}\right)$ of an $N$ cell PV module can be viewed as a partitioning of the PV cell index set $\boldsymbol{A}=\{1,2, \cdots, N\}$. A partitioning is denoted by $n$ subsets $\boldsymbol{B}_{1}, \boldsymbol{B}_{2}, \cdots, \boldsymbol{B}_{n}$, which correspond to $n \mathrm{PV}$ groups consisting of $m_{1}, m_{2}, \cdots, m_{n}$ parallel-connected PV cells, respectively. Therefore, the subsets $\boldsymbol{B}_{1}, \boldsymbol{B}_{2}, \cdots, \boldsymbol{B}_{n}$ should satisfy

$$
\cup_{j=1}^{n} B_{j}=\boldsymbol{A} \text {, }
$$

and

$$
\boldsymbol{B}_{j} \cap \boldsymbol{B}_{k}=\emptyset, \text { for } \forall j, k \in\{1,2, \cdots, n\} \text { and } j \neq k .
$$

Due to the structural characteristics of the reconfigurable PV module architecture shown in Figure 4, we also have

$$
i_{1}<i_{2} \text {, for } \forall i_{1} \in \boldsymbol{B}_{j}, \forall i_{2} \in \boldsymbol{B}_{k} \text { and } 1 \leq j<k \leq n .
$$

A partitioning satisfying (8) (10) is an alphabetical partitioning.

We can develop I-V characteristics of a PV module with the configuration $\mathcal{C}\left(n ; m_{1}, m_{2}, \cdots, m_{n}\right)$. We denote the current and voltage of the $i$-th PV cell by $I_{p v, i}^{c}$ and $V_{p v, i}^{c}$, respectively, the voltage of the $j$-th PV group by $V_{p v, j}^{g}$, the current and voltage of the PV module by $I_{p v}^{m}$ and $V_{p v}^{m}$, respectively. We have

$$
\begin{gathered}
I_{p v}^{m}=\sum_{i \in B_{j}} I_{p v, i}^{c}, \forall j \in\{1,2, \cdots, n\}, \\
V_{p v, j}^{g}=V_{p v, i}^{c}, \text { for } \forall i \in \boldsymbol{B}_{j} \text { and } j \in\{1,2, \cdots, n\},
\end{gathered}
$$

and

$$
V_{p v}^{m}=\sum_{j=1}^{n} V_{p v, j}^{g}
$$

$I_{p v, i}^{c}$ and $V_{p v, i}^{c}$ satisfy the PV cell I-V characteristics (1) (3), given environmental condition $\left(G_{i}, T_{i}\right)$, where $G_{i}$ and $T_{i}$ are the irradiance level and temperature on the $i$-th PV cell, respectively.

\section{PROBLEM STATEMENT}

Figure 5 shows the architecture of a PV system with the string charger interface, which consists of $M$ series-connected reconfigurable PV modules, a charger and a supercapacitor as the EES element. The PV modules share one charger. The string charger interface is cost-effective compared with the individual charger interface, and is widely used. Each PV module in the string has the reconfigurable architecture shown in Figure 4. We restrict the freedom of reconfiguration within each PV module, but it is a reasonable compromise of feasibility and performance.

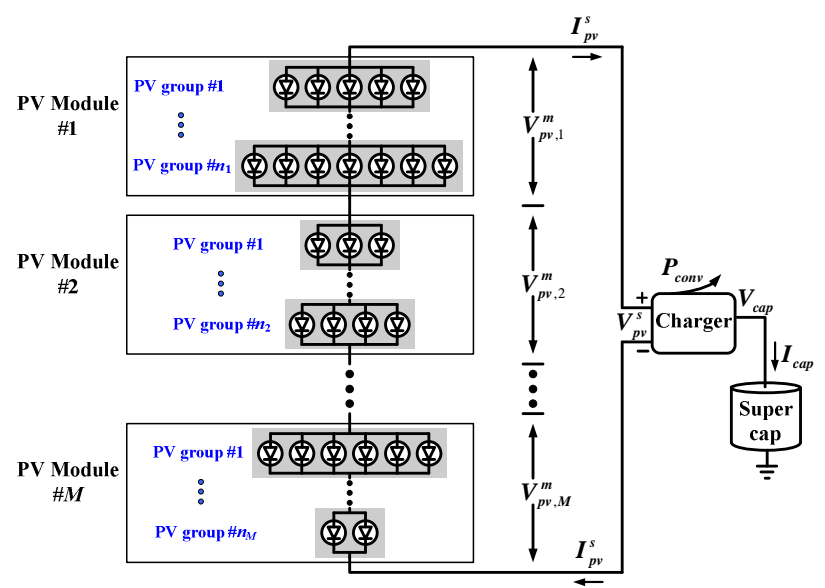

Figure 5. Architecture of a PV system with the string charger interface.

The PV system operation starts at time $T_{0}$ and ends at time $T_{d}$. We use $V_{p v}^{s}(t)$ and $I_{p v}^{s}(t)$ to denote the output voltage and current of the PV module string at $t \in\left[T_{0}, T_{d}\right]$, respectively. We denote the output voltage of the $k$-th $(1 \leq k \leq M)$ PV module by $V_{p v, k}^{m}(t)$. The output currents of the PV modules are the same, namely $I_{p v}^{S}(t)$, and we have

$$
V_{p v}^{s}(t)=\sum_{k=1}^{M} V_{p v, k}^{m}(t) .
$$

We denote the irradiance level and the temperature on the $i$-th $(1 \leq i \leq N) \mathrm{PV}$ cell of the $k$-th $(1 \leq k \leq M) \mathrm{PV}$ module at time $t$ by $G_{k, i}(t)$ and $T_{k, i}(t)$, respectively, and denote the imbalanced configuration of the $k$-th PV module by $\mathcal{C}_{k}(t)$. Obviously $\mathcal{C}_{k}(t)$ $(1 \leq k \leq M)$ are the control variables of the PV reconfiguration algorithm. We assume all PV cell temperatures are the same and constant for the period $\left[T_{0}, T_{d}\right]$, denoted by $T_{p v}$, to focus on the partial shading problem. The PV reconfiguration algorithm proposed in this paper runs in an online manner, i.e., the system controller is not aware of $G_{k, i}(t)(1 \leq k \leq M, 1 \leq i \leq N)$ until time $t$. The relationship of $V_{p v, k}^{m}(t)$ and $I_{p v}^{s}(t)$ depends on $\mathcal{C}_{k}(t)$, $G_{k, i}(t)$, and $T_{k, i}(t)=T_{p v}(i \in\{1,2, \ldots, N\})$, as given in (11) (13). 
We use $V_{c a p}(t)$ and $I_{c a p}(t)$ to denote the terminal voltage and charging current of the supercapacitor, respectively. The power loss of the charger $P_{\text {conv }}(t)$ is determined by its input voltage, input current, output voltage, and output current, i.e., $V_{p v}^{S}(t)$, $I_{p v}^{S}(t), V_{c a p}(t)$, and $I_{c a p}(t)$, respectively, according to (5) (6). According to (4), we also have

$$
V_{p v}^{s}(t) \cdot I_{p v}^{S}(t)=P_{c o n v}(t)+V_{c a p}(t) \cdot I_{c a p}(t) .
$$

The objective of the PV system controller is to find the optimal configuration $\mathcal{C}_{k}^{o p t}(t)(1 \leq k \leq M)$ and the optimal operating point $\left(V_{p v}^{s, o p t}(t), I_{p v}^{s, o p t}(t)\right)$ of the PV module string to maximize $I_{\text {cap }}(t)$ at any time $t \in\left[T_{0}, T_{d}\right]$.

\section{RECONFIGURATION ALGORITHM 5.1 Motivation}

We denote the power at MPP of a PV cell/group/module/string by $M P P$ power. Similarly, there are MPP voltage and MPP current of a PV cell/group/module/string. The utmost solar power harvested by a PV system is the sum of MPP power values of all PV cells. The reconfiguration algorithm aims at making all PV cells operate at or close to their MPPs simultaneously. We update the optimal configurations for PV modules according to current shading patterns to maximize the supercapacitor charging current.
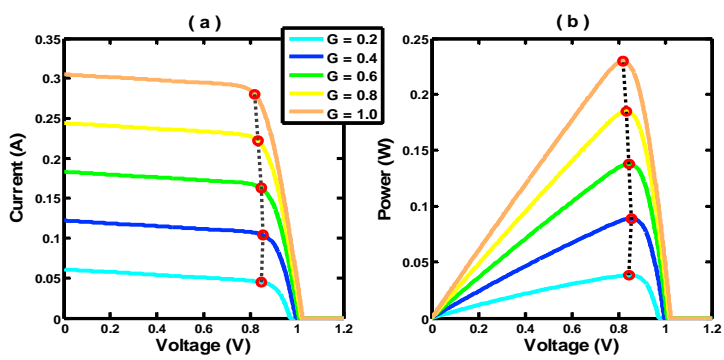

Figure 6. (a) Current-voltage and (b) power-voltage characteristics of a PV cell under different irradiance levels with MPPs labeled by red circles.

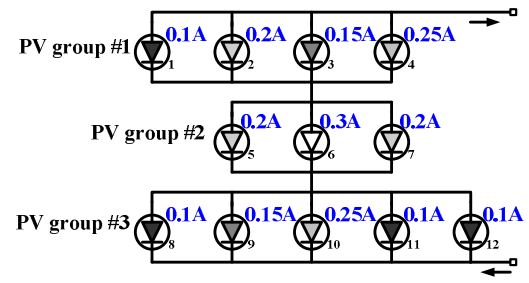

Figure 7. An example of imbalanced reconfiguration according to the PV cell MPP currents at their own MPPs.

One observation made from I-V and P-V characteristics of PV cells in Figure 6 is that MPP voltage values of a PV cell under different irradiance levels are very close to each other, while MPP current values vary significantly. It enables us to use a constant voltage $V_{a v g}^{M P P}$ to approximate MPP voltage. In Figure 7, PV cell MPP current values are calculated from irradiance levels on PV cells and labeled beside PV cells, where the sum of PV cell MPP current values in every PV group is $0.7 \mathrm{~A}$. Then with the configuration in Figure 7, all PV cells can operate close to their own MPPs simultaneously if we set the output voltage of this module at $3 \cdot V_{a v g}^{M P P}$. Similarly, MPP currents of all PV modules in the PV string shall be close to each other, so that the output power of the PV string can be optimized.

\subsection{Algorithm}

We omit time index $t$ for convenience, since the reconfiguration algorithm will be performed at every decision epoch $t \in\left[T_{0}, T_{d}\right]$. We denote the MPP voltage and current values of the $i$-th $(1 \leq i \leq N) \mathrm{PV}$ cell in the $k$-th $(1 \leq k \leq M) \mathrm{PV}$ module by $V_{p v, k, i}^{c, M P P}$ and $I_{p v, k, i}^{c, M P P}$, respectively, which are calculated from the condition $\left(G_{k, i}, T_{p v}\right)$. These $V_{p v, k, i}^{c, M P P}$ and $I_{p v, k, i}^{c, M P P}$ values are inputs of our reconfiguration control algorithm. All $V_{p v, k, i}^{c, M P P}$ can be approximated by $V_{a v g}^{M P P}$ due to the observation in Section 5.1. The objective of our algorithm is finding the optimal configurations $\mathcal{C}_{k}^{o p t}\left(n_{k} ; m_{k, 1}, m_{k, 2}, \cdots, m_{k, n_{k}}\right)(1 \leq k \leq M)$. It consists of an initial allocation procedure and a kernel algorithm. The initial allocation procedure determines the number of PV groups $n_{k}$ for each $k$-th PV module. The kernel algorithm finds the optimal $m_{k, 1}$, $m_{k, 2}, \ldots, m_{k, n_{k}}$ values corresponding to the optimal alphabetical partitioning $\boldsymbol{B}_{k, 1}, \boldsymbol{B}_{k, 2}, \cdots, \boldsymbol{B}_{k, n_{k}}$ of the set $\boldsymbol{A}=\{1,2, \cdots, N\}$ for each $k$-th PV module, given $I_{p v, k, i}^{c, M P P}(1 \leq i \leq N)$ and $n_{k}$.

The initial allocation procedure determines the number of PV groups $n_{k}$ for each $k$-th PV module in a one shot manner. It can improve PV system output power due to the following reasons:

(1) The MPP currents of PV modules in the PV string should be close to each other.

(2) The MPP voltage of the PV module string should match supercapacitor voltage to reduce power loss in the charger. We do not look into detailed configuration of each PV module in the initial allocation procedure. Detailed procedures of the initial allocation are described as follows. First, we use $\widehat{P}_{p v}^{s, M P P}$ to denote the sum of MPP power values of all PV cells in the PV module string, given by

$$
\hat{P}_{p v}^{s, M P P}=\sum_{1 \leq k \leq M} \sum_{1 \leq i \leq N} V_{p v, k, i}^{c, M P P} \cdot I_{p v, k, i}^{c, M P P} .
$$

$\hat{P}_{p v}^{s, M P P}$ is an optimistic estimation of the MPP power of the whole PV module string. We use $\hat{P}_{p v, k}^{m, M P P}$ to denote the sum of MPP power values of all PV cells in the $k$-th PV module, given by

$$
\hat{P}_{p v, k}^{m, M P P}=\sum_{1 \leq i \leq N} V_{p v, k, i}^{c, M P P} \cdot I_{p v, k, i}^{c, M P P} .
$$

It is an optimistic estimation of MPP power of the $k$-th PV module.

Next we find the estimated optimal PV string voltage and current, denoted by $\hat{V}_{p v}^{s, o p t}$ and $\hat{I}_{p v}^{s, o p t}$, respectively, satisfying $\hat{V}_{p v}^{s, o p t} \cdot \hat{I}_{p v}^{s, o p t}=\hat{P}_{p v}^{s, M P P}$, so that the estimated supercapacitor charging current, denoted by $\hat{I}_{c a p}^{o p t}$, is maximized. We have:

$$
\begin{aligned}
& \left(\hat{V}_{p v}^{s, o p t}, \hat{I}_{p v}^{s, o p t}\right)=\underset{V_{p v}^{s} \cdot I_{p v}^{S}=\hat{P}_{p v}^{s, M P P}}{\arg \max } \operatorname{Chg} g_{-} \text {Out_I } I\left(V_{p v}^{s}, I_{p v}^{s}, V_{c a p}\right), \\
& \hat{I}_{c a p}^{o p t}=\max _{V_{p v}^{s} \cdot I_{p v}^{S}=\hat{P}_{p v}^{s, M P P}} \text { Chg_Out_I }\left(V_{p v}^{s}, I_{p v}^{s}, V_{c a p}\right) \text {, }
\end{aligned}
$$

where the function $\mathrm{Chg}_{-} \mathrm{Out} I(\mathrm{I})$ is defined in Section 2.2.

Next we find a proper range of the estimated PV string output voltage $\left[\hat{V}_{p v}^{s, \min }, \hat{V}_{p v}^{s, \max }\right]$, so that for any $\left(V_{p v}^{s}, I_{p v}^{s}\right)$ pair satisfying $V_{p v}^{s} \in\left[\hat{V}_{p v}^{s, \min }, \hat{V}_{p v}^{s, \max }\right]$ and $V_{p v}^{s} \cdot I_{p v}^{s}=\hat{P}_{p v}^{s, M P P}$, the supercapacitor charging current calculated through Chg_Out_I $\left._{\text {o }} V_{p v}^{s}, I_{p v}^{s}, V_{c a p}\right)$ is within a small range of $\hat{I}_{\text {cap }}^{o p t}$, i.e.,

$$
\begin{gathered}
\max _{V_{p v}^{s} \in\left[\widehat{V}_{p v}^{s, m i n}, \hat{V}_{p v}^{s, m a x}\right], V_{p v}^{s} \cdot I_{p v}^{s}=\hat{P}_{p v}^{s, M P P}} \text { Chg_Out_I } \\
\geq(1-\varepsilon) \hat{I}_{p v}^{s}, I_{p v p}^{s},
\end{gathered}
$$

where $\varepsilon$ is a predefined small value. Furthermore we calculate the range of the estimated number of PV groups in the PV module string $\left[\hat{n}_{\text {total }}^{\min }, \hat{n}_{\text {total }}^{\max }\right]$, where $\hat{n}_{\text {total }}^{\min }$ and $\hat{n}_{\text {total }}^{\max }$ satisfy 


$$
\hat{n}_{\text {total }}^{\min }=\left\lceil\frac{\widehat{V}_{p v}^{s, m i n}}{V_{\text {avg }}^{M P P}}\right\rceil, \quad \hat{n}_{\text {total }}^{\max }=\left\lfloor\frac{\widehat{V}_{p v}^{s, \max }}{V_{\text {avg }}^{M P P}}\right\rfloor .
$$

For each possible estimated number of total PV groups in the PV string $\hat{n}_{\text {total }}^{(l)}\left(\hat{n}_{\text {total }}^{(l)} \in\left[\hat{n}_{\text {total }}^{\min }, \hat{n}_{\text {total }}^{\max }\right]\right.$ and $1 \leq l \leq \hat{n}_{\text {total }}^{\max }-$ $\left.\hat{n}_{\text {total }}^{\text {min }}+1\right)$, we calculate the possible numbers of $P V$ groups for each $P V$ module. To reduce the exploration space, we assume that there are only two possible numbers of PV groups for each $k$-th PV module $n_{k}^{(l),-}$ and $n_{k}^{(l),+}$, respectively, given by

$$
n_{k}^{(l),-}=\left\lfloor\frac{\hat{n}_{\text {total }}^{(l)} \cdot \hat{P}_{p v, k}^{m, M P P}}{\hat{P}_{p v}^{S, M P P}}\right\rfloor, \quad n_{k}^{(l),+}=n_{k}^{(l),-}+1 .
$$

The underlying principle of (22) is that MPP currents of different PV modules in the PV string should be close to each other. We define a vector variable $\boldsymbol{n}^{(l)}=\left(n_{1}^{(l)}, n_{2}^{(l)}, \ldots, n_{M}^{(l)}\right)$, in which $n_{k}^{(l)}$ $(1 \leq k \leq M)$ denotes the possible number of $\mathrm{PV}$ groups in the $k$ th PV module. There are $2^{M}$ (vector) values $\boldsymbol{n}^{(l)}$ could assume, since $n_{k}^{(l)}$ is either $n_{k}^{(l),-}$ or $n_{k}^{(l),+}$. Hence, we can find the optimal $\boldsymbol{n}^{(l)}$ among all possible $l$ and $n_{k}^{(l)}(1 \leq k \leq M)$, such that the estimated supercapacitor charging current

$$
\text { Chg_Out_I }\left(\sum_{1 \leq k \leq M} n_{k}^{(l)} \cdot V_{a v g}^{M P P}, \min _{1 \leq k \leq M}\left(\frac{\hat{P}_{p v, k}^{m, M P P}}{n_{k}^{(l)} \cdot V_{a v g}^{M P P}}\right), V_{c a p}\right) \text {, }
$$

can be maximized, i.e., the optimization objective would be

$$
\max _{l, \boldsymbol{n}(l)} \text { Chg_Out_I }\left(\sum_{1 \leq k \leq M} n_{k}^{(l)} \cdot V_{a v g}^{M P P}, \min _{1 \leq k \leq M}\left(\frac{\hat{P}_{p v, k}^{m, M P P}}{n_{k}^{(l)} \cdot V_{a v g}^{M P P}}\right), V_{c a p}\right) \text {. }
$$

Then we assign the number of PV groups in each $k$-th PV module $n_{k}$, to be the value $n_{k}^{(l)}$ in the optimal vector $\boldsymbol{n}^{(l)}$. An outline of the initial allocation procedure is shown in Algorithm 1.

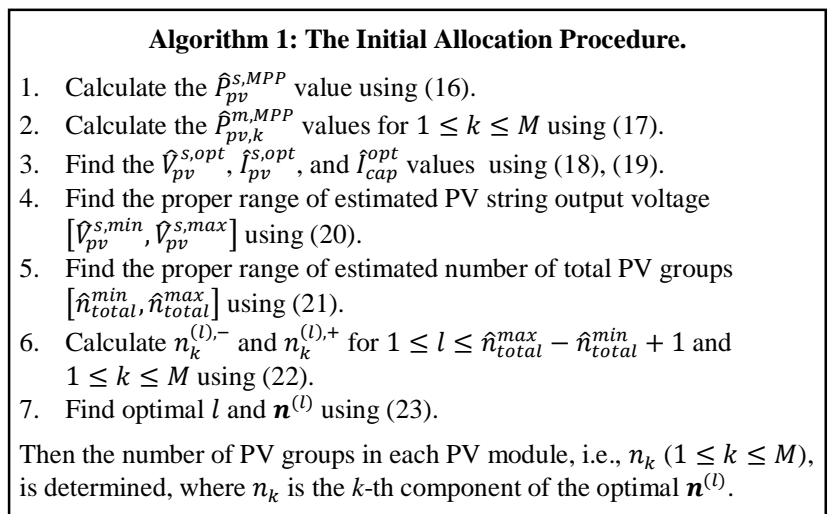

After the initial allocation procedure, the kernel algorithm is applied once to each PV module with given $n_{k}$. The kernel algorithm finds the optimal $m_{k, 1}, m_{k, 2}, \ldots, m_{k, n_{k}}$ values for the $k$ th PV module. We use $\hat{I}_{p v, k, j}^{g, M P P}$ to denote the sum of PV cell MPP currents in the $j$-th PV group of the $k$-th PV module, given by

$$
\hat{I}_{p v, k, j}^{g, M P P}=\sum_{i \in B_{k, j}} I_{p v, k, i}^{c, M P P} .
$$

$\hat{I}_{p v, k, j}^{g, M P P}$ is an estimation of MPP current of the $j$-th PV group in the $k$-th PV module. Inspired by motivations stated in Section 5.1, we should make all $\hat{I}_{p v, k, j}^{g, M P P}$ values $\left(1 \leq j \leq n_{k}\right)$ as close to each other as possible. Equivalently, we maximize the minimal $\hat{I}_{p v, k, j}^{g, M P P}$ value of the $k$-th PV module, denoted by Min_Sum_I ${ }_{k}$, since MPP current of the $k$-th PV module is restricted by that minimal value. The objective of kernel algorithm is finding optimal alphabetical partitioning $\boldsymbol{B}_{k, 1}, \boldsymbol{B}_{k, 2}, \cdots, \boldsymbol{B}_{k, n_{k}}$ of the $k$-th PV module, so that Min_Sum_I $I_{k}$ is maximized, i.e., the objective would be $\max _{\boldsymbol{B}_{k, 1}, \boldsymbol{B}_{k, 2}, \cdots, \boldsymbol{B}_{k, n_{k}}}$ Min_Sum_I $I_{k}=\max _{\boldsymbol{B}_{k, 1}, \boldsymbol{B}_{k, 2}, \cdots, \boldsymbol{B}_{k, n_{k}}} \min _{1 \leq j \leq n_{k}} \hat{I}_{p v, k, j}^{g, M P P}$.

Then all PV cells in the $k$-th PV module under the configuration corresponding to the optimal alphabetical partitioning $\boldsymbol{B}_{k, 1}, \boldsymbol{B}_{k, 2}$, $\cdots, \boldsymbol{B}_{k, n_{k}}$ could work very close to their MPPs, if the $k$-th PV module output voltage is set at $n_{k} \cdot V_{a v g}^{M P P}$.

Consider a general problem of finding optimal configuration for an $l_{1}$-cell $\left(l_{1} \leq N\right.$, corresponding to the first $l_{1}$ cells of the original $N$ cells in the $k$-th PV module) PV module composed of $l_{2}\left(l_{2} \leq n_{k}\right) \mathrm{PV}$ groups, given $I_{p v, k, i}^{c, M P P}\left(1 \leq i \leq l_{1}\right)$ values and $l_{2}$. This is equivalent to finding the optimal alphabetical partitioning $\boldsymbol{B}_{k, 1}^{l_{1}, l_{2}}, \boldsymbol{B}_{k, 2}^{l_{1}, l_{2}}, \cdots, \boldsymbol{B}_{k, l_{2}}^{l_{1}, l_{2}}$ of the set $\boldsymbol{A}^{l_{1}}=\left\{1,2, \cdots, l_{1}\right\}$, which is optimal in that the value Min_Sum_ $I_{k}^{l_{1}, l_{2}}=\min _{1 \leq j \leq l_{2}} \sum_{i \in B_{k, j}^{l_{1}, l_{2}}} I_{p v, k, i}^{c, M P P}$ is maximized. We call this problem $\left(l_{1}, l_{2}\right)$ reconfiguration problem. When $l_{1}=N$ and $l_{2}=n_{k}$, the $\left(l_{1}, l_{2}\right)$ reconfiguration problem becomes the original reconfiguration problem of the $k$-th PV module as stated in (25). We find optimal substructure property of $\left(l_{1}, l_{2}\right)$ reconfiguration problem below, implying the applicability of dynamic programming.

The optimal substructure observation: Suppose that $\left(l_{1}, l_{2}\right)$ reconfiguration problem has been optimally solved, and that the last $\left(l_{2}\right.$-th) PV group consists of $m_{k, l_{2}}^{l_{1}, l_{2}}$ PV cells. Then the subproblem of finding the optimal configuration for the first $l_{1}-$ $m_{k, l_{2}}^{l_{1}, l_{2}} \mathrm{PV}$ cells within $l_{2}-1 \mathrm{PV}$ groups, which corresponds to the $\left(l_{1}-m_{k, l_{2}}^{l_{1}, l_{2}}, l_{2}-1\right)$ reconfiguration problem, has to be solved optimally. From this observation, we have Algorithm 2 based on dynamic programming as the kernel algorithm for finding the optimal configuration for the $k$-th PV module with given $n_{k}$.

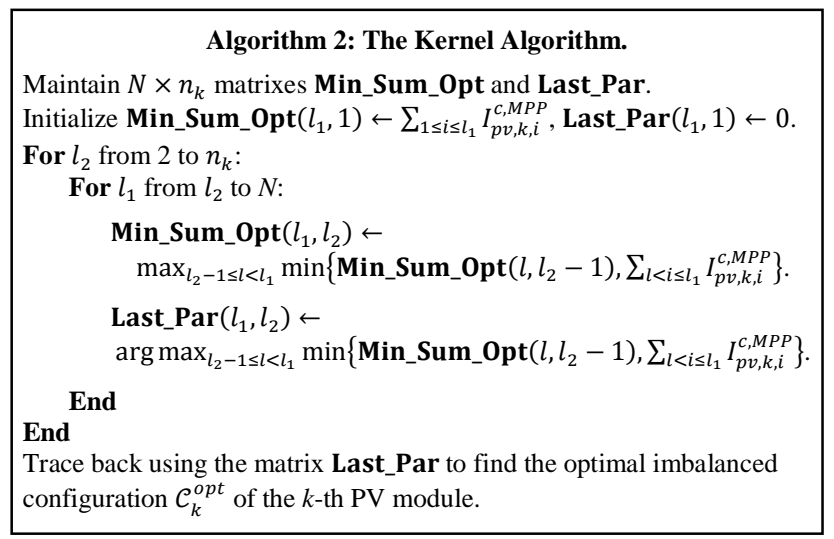

\section{EXPERIMENTAL RESULTS}

We compare performances of the PV system with reconfiguration technique and the baseline system without reconfiguration. Both systems consist of $3 \mathrm{PV}$ modules $(M=3)$ with $60 \mathrm{PV}$ cells each $(N=60)$, a charger and a supercapacitor. PV modules in the proposed system have the reconfigurable architecture in Figure 4, while PV modules in the baseline system have a fixed 10x6 configuration, where $10 \mathrm{PV}$ groups are series-connected with 6 PV cells per PV group. The charger model is Linear Technology LTM4607 converter, and the supercapacitor is $100 \mathrm{~F}$. In the baseline system, we incorporate improved MPPT technique [5] and bypass diodes with PV cells [7] to enhance power output under partial shading. In the proposed system, we incorporate the MPTT technique to feed maximum power into the supercapacitor. 
First we test instantaneous power output level of the two systems under the shading pattern shown in Figure 8. For the proposed system, Figure 8 shows physical locations of PV cells in the $3 \mathrm{PV}$ modules, instead of the real imbalanced configuration of PV modules. Table 1 summarizes the power output improvement of the proposed system compared to the baseline system, given the shading pattern in Figure 8 and the $V_{\text {cap }}$ values. As shown in Table 1, the proposed system achieves up to a $2.31 \mathrm{X}$ output power improvement compared with the baseline system with a $V_{\text {cap }}$ of 45 $\mathrm{V}$ demonstrating effectiveness of the reconfiguration technique.
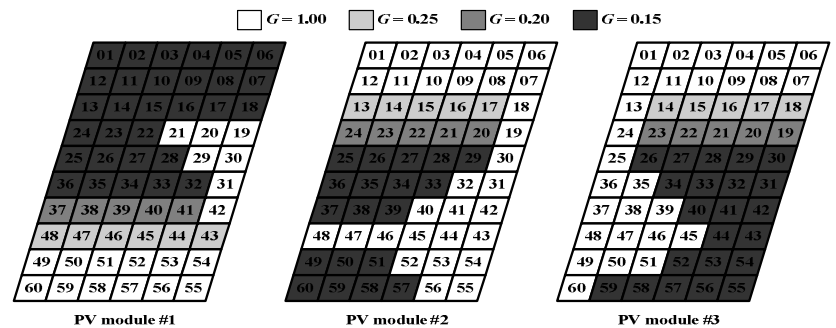

Figure 8. The shading pattern on the 3-module PV string.

Table 1. The improvement of instantaneous power level of the PV system with the imbalanced reconfiguration technique.

\begin{tabular}{|c|c|c|c|}
\hline$V_{\text {cap }}(\mathrm{V})$ & 5 & 25 & 45 \\
\hline $\begin{array}{l}\text { Power output } \\
\text { improvement }\end{array}$ & $1.91 \mathrm{X}$ & $2.04 \mathrm{X}$ & $2.31 \mathrm{X}$ \\
\hline $\begin{array}{l}\text { PV module \#1 } \\
\text { configuration }\end{array}$ & $\mathcal{C}(3 ; 30,22,8)$ & $\mathcal{C}(6 ; 20,10,16,6,4,4)$ & $\mathcal{C}(4 ; 28,18,8,6)$ \\
\hline $\begin{array}{l}\text { PV module \#2 } \\
\text { configuration }\end{array}$ & $\mathcal{E}(4 ; 9,21,15,15)$ & $\begin{array}{c}\mathcal{C}(9 ; 4,4,4,11,9,10 \\
4,7,7)\end{array}$ & $\mathcal{E}(6 ; 6,6,18,12,6,12)$ \\
\hline $\begin{array}{l}\text { PV module \#3 } \\
\text { configuration }\end{array}$ & $(4 ; 8,16,21,15)$ & $\begin{array}{c}\mathcal{C}(8 ; 4,4,4,12,12,9 \\
4,11)\end{array}$ & $\mathcal{C}(5 ; 6,6,23,11,14)$ \\
\hline
\end{tabular}

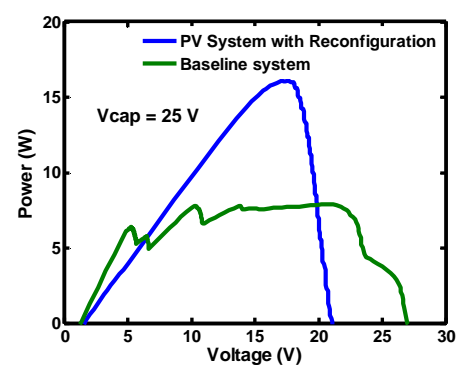

Figure 9. P-V characteristics of the PV strings in the PV system with imbalanced reconfiguration and baseline system.

Table 1 also provides near-optimal configuration obtained by the reconfiguration control algorithm for each PV module in the $\mathrm{PV}$ string. Figure 9 plots the P-V curves of the PV module strings in the two systems with the shading pattern in Figure 8 and a $V_{\text {cap }}$ of $25 \mathrm{~V}$. The proposed system achieves a peak power much higher than that of the baseline system. Moreover our PV reconfiguration technique makes $\mathrm{P}-\mathrm{V}$ curve of the proposed system unimodal under partial shading, while P-V curve of the baseline system has multiple peaks. Therefore standard MPTT technique can be incorporated into the proposed system without extra modifications. But in baseline system, the MPPT technique must be modified to track a global optimal operating point instead of a local one.
We also test the overall efficiency of the two systems in a time period of 300 minutes, with timing parameters $T_{0}=0 \mathrm{~min}$ and $T_{d}=300 \mathrm{~min}$. About $2 / 3$ of all PV cells in the $3 \mathrm{PV}$ modules are shaded and irradiance levels on these shaded cells are randomly generated within the range $[0,0.5]$ which also change with time. The proposed system updates its module configurations once per minute according to current shading pattern and charger efficiency variation. We compare the electrical energy stored into the supercapacitors in the two systems during time $\left[T_{0}, T_{d}\right]$, and the proposed system achieves a $1.59 \mathrm{X}$ improvement compared to the baseline system, demonstrating that the reconfiguration technique can effectively combat the partial shading effects.

\section{CONCLUSION}

This paper addresses the power output loss problem of a PV system with the string charger interface under partial shading. We introduce the imbalanced reconfiguration technique to combat the partial shading effects. We propose the imbalanced PV cell connection topology for the first time and also provide an effective reconfiguration control algorithm, which realizes adaptive and near-optimal PV module reconfiguration for each PV module in the PV string according to the partial shading pattern and the charger efficiency variation. The proposed reconfiguration control algorithm is based on dynamic programming with polynomial time complexity.

\section{ACKNOWLEDGEMENT}

This work is sponsored in part by a grant from the National Science Foundation, and by the Brain Korea 21 Project and MidCareer Researcher Program through NRF Grant funded by the MEST (No. 2011-0016480). The ICT at Seoul National University provides research facilities for this study.

\section{REFERENCES}

[1] N. Femia, G. Petrone, G. Spagnuolo, and M. Vitelli, "Optimization of perturb and observe maximum power point tracking method," IEEE T. on Power Electronics, 2005.

[2] F. Liu, S. Duan, F. Liu, B. Liu, and Y. Kang, "A variable step size INC MPPT method for PV systems," IEEE T. on Industrial Electronics, 2008.

[3] Y. Kim, N. Chang, Y. Wang, and M. Pedram, "Maximum power transfer tracking for a photovoltaic-supercapacitor energy system," in ISLPED, 2010

[4] C. Lu, V. Raghunathan, and K. Roy, "Maximum power point considerations in micro-scale solar energy harvesting systems," in IEEE ISCAS, 2010

[5] H. Patel, and V. Agarwal, "Maximum power point tracking scheme for PV systems operating under partially shaded conditions," IEEE T. on Industrial Electronics, 2008.

[6] R. Bruendlinger, B. Bletterie, M. Milde, and H. Oldenkamp, "Maximum power point tracking performance under partially shaded PV array conditions," in Proc. $21^{\text {st }}$ EUPVSEC, 2006.

[7] D. Nguyen, and B. Lehman, "An adaptive solar photovoltaic array using model-based reconfiguration algorithm," IEEE T. on Industrial Electronics, 2008.

[8] G. Velasco-Quesada, F. Guinjoan-Gispert, R. Pique-Lopez, M. Roman-Lumbreras, and A. Conesa-Rosa, "Electrical PV array reconfiguration strategy for energy extraction improvement in gridconnected PV systems," IEEE T. on Industrial Electronics, 2009.

[9] M. A. Chaaban, M. Alahmad, J. Neal, J. Shi, C. Berryman, Y. Cho, S. Lau, H. Li, A. Schwer, Z. Shen, J. Stansbury, and T. Zhang, "Adaptive photovoltaic system," in IECON, 2010.

[10] Y. Kim, S. Park, Y. Wang, Q. Xie, N. Chang, M. Poncino, and M Pedram, "Balanced reconfiguration of storage banks in a hybrid electrical energy storage system," in ICCAD, 2011. 
[11] W. Lee, Y. Kim, Y. Wang, N. Chang, M. Pedram, and S. Han,

"Versatile high-fidelity photovoltaic module emulation system," in ISLPED, 2011.
[12] Y. Wang, Y. Kim, Q. Xie, N. Chang, and M. Pedram, "Charge migration efficiency optimization in hybrid electrical energy storage (HEES) systems," in ISLPED, 2011. 